\title{
MY APPROACH to Choosing Ventricular Pacing Sites in Patients With Severe Heart Failure*
}

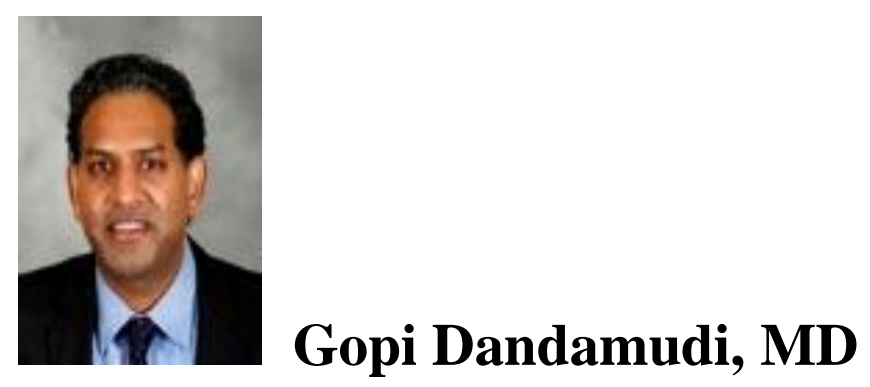

Patients with severe heart failure (HF) present a unique challenge when it comes to chronic ventricular pacing (VP). Chronic right VP can potentially have long-term detrimental effects likely related to pacing-induced dyssynchrony, including worsening cardiomyopathy and increasing the risk for atrial fibrillation. Algorithms have been developed over time to reduce VP by extending AV delays to non-physiological levels. Biventricular pacing (BiV) aims to avoid pacing-induced dyssynchrony and mitigate some of these adverse effects of right VP. However, recent trials have shown equivocal results, especially in patients with mildly reduced ejection fractions (EF). I usually divide patients into three categories:

Patients with prophylactic devices such as ICDs in whom pacing is not indicated

In this situation, I place the lead on the anterior septum to ensure that the ICD coil is across the tricuspid valve in its entirety. The lead serves its purpose as a shocking coil with backup pacing and for anti-tachycardia pacing.

Patients with devices in whom pacing is required (all patients with different degrees of heart blocks, QRS durations < $\mathbf{1 5 0}$ ms, and HF symptoms with indications for biventricular pacing)

In these cases, I attempt permanent His-bundle pacing (HBP). I believe it is the only true physiological form of pacing, and, in a vast majority of cases, the His bundle can be recruited reliably and chronically, even in patients with infranodal block. I have considerable experience and long-term follow-up data to support this form of pacing. Other clinicians have also been adopting this strategy more recently. In rare cases, I do place a backup pacing lead in the right ventricular septum if the implanting HBP thresholds are higher than anticipated. 


\section{Patients with wide left bundle branch blocks (LBBB)}

This cohort still presents challenges when it comes to pacing strategies. BiV pacing has been shown to improve morbidity and mortality; however, response rates are still less than ideal, with only two-thirds of patients responding to it. Recruitment of LBBB with HBP has been shown to be feasible since the early 1970s. I have successfully performed this procedure in patients with chronic LBBB who have shown dramatic responses from an HF symptom standpoint. I usually discuss these two options with all my patients before proceeding with permanent pacing. In patients with severe HF symptoms, I still implant left ventricular leads as my first approach. If lead placement is suboptimal due to lead location, left phrenic nerve issues, or high pacing thresholds, I then attempt HBP. In patients with less severe HF symptoms, I attempt HBP first if the patient is agreeable to it. In my clinical practice, I have yet to have a single patient turn down HBP in favor of LV pacing.

In my opinion, permanent HBP allows a unique opportunity to offer true physiological pacing. It is what evolution has selected over time to be the most efficient way to activate the ventricles, and replicating this physiology has to be at least as effective if not superior to other forms of pacing.

Program Director, Indiana University Health Atrial Fibrillation Center, Clinical Assistant Professor of Medicine, Indiana University School of Medicine, Indianapolis, IN. email: gdandamu@iu.edu

*First published on PracticeUpdate on October 03, 2016. Republished with permission. 